\section{Correspondence}

https://doi.org/10.11646/zootaxa.4999.4.9

http://zoobank.org/urn:lsid:zoobank.org:pub:29C86265-7073-4D5D-A03B-BF18048CE5F0

\title{
Updates on the Fixator-Facilitating the investigation, mounting, and photography of structures and specimens in liquid
}

\author{
DOMINIC WANKE ${ }^{1,2^{*}}$, JONAH M ULMER $^{2,3}$, INGO WENDT $^{2,4} \&$ HOSSEIN RAJAEI $^{2,5}$ \\ ${ }^{1}$ University of Hohenheim, Systematic Entomology (190n), Garbenstr. 30, D-70599 Stuttgart, Germany \\ ${ }^{2}$ State Museum of Natural History Stuttgart, Entomology, Rosenstein 1, D-70191 Stuttgart, Germany \\ ${ }^{3}$ "="jonah.ulmer@smns-bw.de; @ ittps://orcid.org/0000-0002-9185-6378 \\ 4!"ingo.wendt@smns-bw.de; 이ttps://orcid.org/0000-0002-8367-4048 \\ 5 "Eossein.rajaei@smns-bw.de; @ https://orcid.org/0000-0002-3940-3734

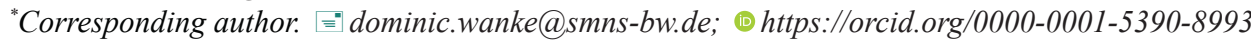

The investigation, imaging, description and interpretation of morphological characters is essential in taxonomy (Padial et al. 2010, Montesanto 2015). Examination of internal genitalia structures often require dissection in liquid, resulting in genitalia stored in microvials or embedded on permanent slides (Gurney et al. 1964, Robinson 1976, Ungureanu 1972). Character drawings and photographs in different or natural positions are mandatory for a precise scientific illustration and description (Wanke \& Rajaei 2018). This step potentially presents a challenge for taxonomists as structures drift or move during investigation or stacking photography (Su 2016). Moreover, evaporation of ethanol and vibrations challenge the patience of the researcher (Su 2016, Haselböck et al. 2018). To overcome the most common struggles different ways have been published, either using viscose liquids (e.g. hand sanitizer, agarose gel) or weak adhesives (e.g. boric acid ointment) or in some cases a mechanical fixation (Gurney et al. 1964, Su 2016, Haselböck et al. 2018, Wanke \& Rajaei 2018, Wanke et al. 2019). Recently, these mechanical fixators have been shown to be a useful tool during the investigation and documentation of structures through various groups of arthropods (e.g. Müller 2021, Rajaei et al. 2021). In several studies on Lepidoptera, the Fixator facilitated the photography of genitalia structures, as it allowed for easy comparative analysis (Wanke et al. 2020a; Wanke et al. 2020b). Though in some cases modifications are needed. These include a stronger and highly durable connection between petri dish and nylon thread holders, as well as the ability to vary the height of the nylon thread. The latter is important if, additionally, a translucent and soft underlay with anti-slip properties is needed. Here we present several modifications of the Fixator and illustrate its advantages via the imaging of the spermatheca in Augacephalus ezendami (Gallon, 2001), a theraphosid spider distinguished from congeners by the cross section of the spermatheca (Gallon 2010).

\section{Stepwise guide for building of the updated Fixator}

Material: Plastic Petri dish, five M3 nylon hex cylinder head screws, five M3 nylon hex sleeve nuts, nylon thread, a razor blade, super glue (based on Ethyl cyanoacrylate (ECA)), thread seal tape (PTFA tape). Additionally, if desired silicone rubber (SILIXON10 formerly known as EROSIL10) or a serrated piece of plastic as an underlay.

1. Drill four holes equidistance from one another in a "+" pattern in the bottom of the Petri dish. Spacing of the holes is dependent upon screw size and size of the Petri dish (Fig. 1A-B). One additional hole is drilled in the wall of the Petri dish perpendicular to one of the previously drilled holes (Fig. 1A). Note: In the provided example we used a 55 $\mathrm{mm}$ Petri dish and found placement of the holes $10 \mathrm{~mm}$ from the walls to be optimal. Too close to the walls made it difficult to thread the nylon through the modified screws and too close to the center can cause the screw heads to cast a shadow on the working stage during imaging.

2. Take two screws and drill a hole fully through the side of the heads, use a drill bit slightly larger than the diameter of the nylon thread (Fig. 1C). The height of the holes in the screw head will determine the height of the thread across the working stage (see Fig. 1C h) (i.e. for small objects a hole closer to the base is recommended). Note that if a silicon underlay is desired the holes will need to be drilled slightly higher to account for the thickening of the base from the silicon. 
3. The screws modified in step 2 are placed in the holes which are linear with the hole previously drilled in the side of the casing. The two unprepared screws are inserted into the remaining two holes (Fig. 1D) (note: if a plastic underlay is desired go to point $8 \mathrm{~A}$ and then continue with 4).

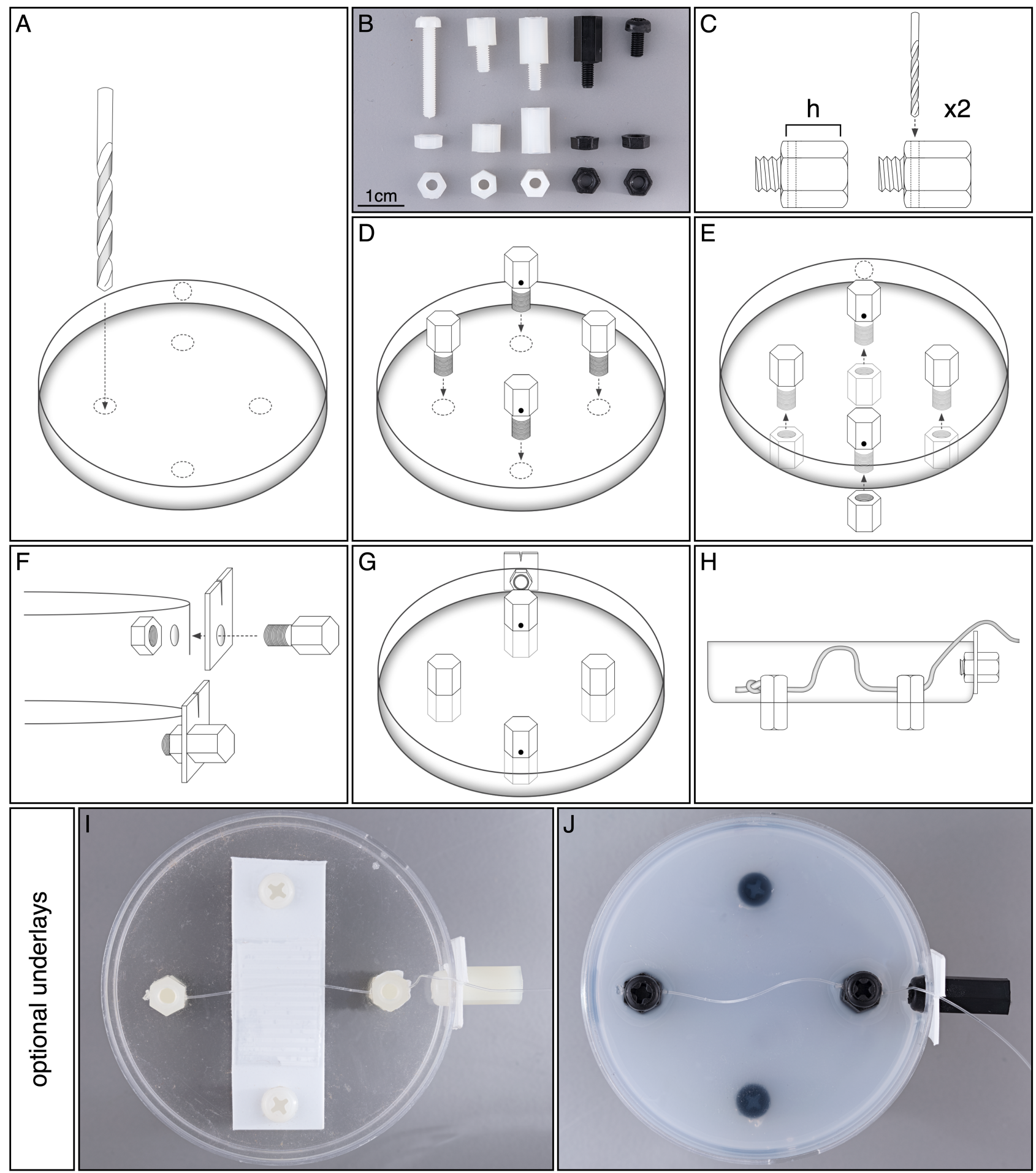

FIGURE 1. Construction of the updated Fixator. A. Drilling of the holes (four in the bottom and one in the wall) in the desired spacing. B. Selection of screw and nut types which can be used in construction. C. Drill a hole in the side of two screw heads (as nylon thread holders). D. Insert the prepared screws to align to the hole in the wall, the others in the remaining holes. E. Secure screws to the Petri dish with thread seal tape and nuts F. Secure the plastic piece to the wall via a screw with thread seal tape and nut in the previously drilled hole. G. Ensure the modified screws and plastic are aligned. H. Thread nylon through the modified screw head holes securing with a knot on one end and via the plastic slit on the other. I. Fixator showing plastic underlay. J. Fixator with silicone underlay. 
4. Wrap all of the screw threads in thread seal tape before fastening to the Petri dish with the nylon sleeve nuts. Super glue can also be used in place of thread seal tape.

5. A small piece of plastic is taken and a hole (of equal size to the nylon screws diameter) is drilled through it. A small slit is cut on one edge of the plastic, ensuring not to cut through to the screw hole previously drilled. The plastic piece is fitted onto a screw with the slit on the top edge and inserted into the hole drilled into the wall of the petri dish in step 1. The screw is secured with thread seal tape and a nut (Fig. 1F-G).

6. A simple overhand knot is tied in one end of the nylon thread. The thread is then passed through the holes in the modified screws (Fig. 1H). The thread is then passed through the slit in the plastic to provide tension. Like in the previous version the knot can be glued to its holder.

7. The Fixator without underlay is now ready to use, the strength of the fixation can be adjusted by pulling the nylon thread

8. If an underlay is desired, the type should be chosen. Here we present two types of underlays, a serrated plastic one $(8 \mathrm{~A})$ and a translucent and soft silicon underlay (8B).

8A. For the plastic underlay any plastic piece, with or without structure, can be used. Here we used a white plastic base with a serrated plastic piece glued on top. For its mounting within the Petri dish, holes equal in size to the screws diameter need to be drilled in the plastic, ensuring the distance is equal to that of the screw holes within the dish. It is then secured to the plate via the unmodified screws perpendicular to the nylon thread (Fig. 1I).

8B. For the translucent and soft underlay, we chose silicone rubber (SILIXON10 formerly known as EROSIL10). The silicone should be mixed according to package directions. Once prepared, it can be poured into the previously built Fixator. Ensure the nylon thread has slack and is held above the dish so it is not submerged once the silicone is poured in. The petri dish should be filled to slightly higher than the holes in the modified screws which hold the nylon thread. This ensures the thread will lay flush against the surface. Allow to sit and solidify for the time specified on the packaging and removed any excess silicone from the dish or nylon thread (Fig. 1J). The silicone can safely be dyed any colour during the initial mixing if the natural opaque white is not preferred. The silicone can also be replaced after heavy usage by peeling it away from the Petri dish and pouring in new mixture.

\section{Fixation of a theraposid spermathecae to access distal cross-sections}

Cross sections of the spermathecae of Theraphosidae are a useful character to distinguish species (e.g. Gallon 2010). Using the updated Fixator with silicon underlay, spermathecae can easily be fixated in positions enabling dorsal and distal observations (Fig. 2). The receptacles are not required to be removed from the ventral cuticula, thereby reducing damage to the specimen or loss of these often small tissue samples. By varying the pressure of the nylon thread on the cuticle and the silicone underlay the spermatheca can be positioned allowing the observation and imaging of its cross-section and hence a more standardized imaging of these structures.
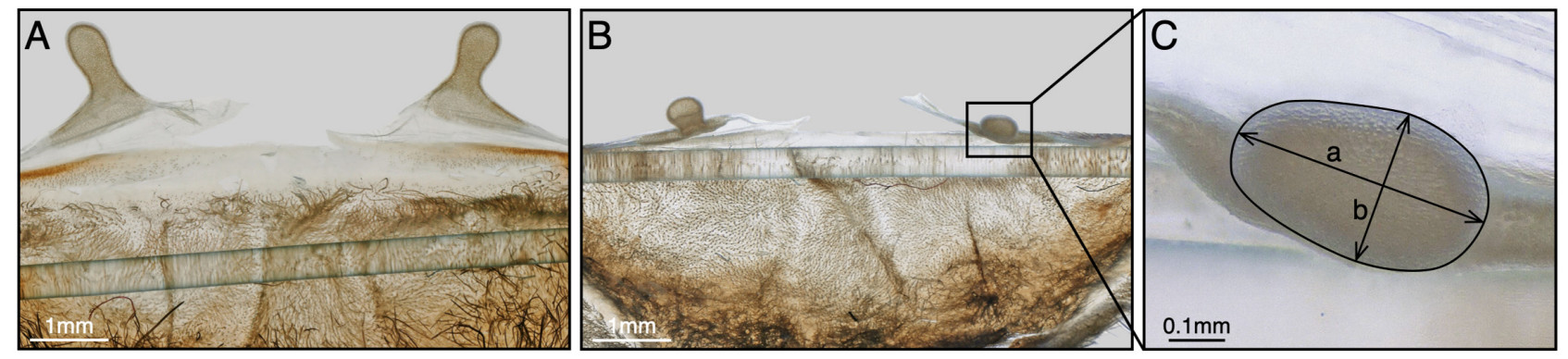

FIGURE 2. Spermatheca of Augacephalus ezendami (Gallon, 2001) (accession number: SMNS-Aran-001543) in dorsal view (A), distal view (B) and close-up on (C). Line $a=437.85 \mu \mathrm{m}, \mathrm{b}=260.75 \mu \mathrm{m}$.

\section{Acknowledgements}

We are grateful to the subject editor of Zootaxa Reza Zahiri (Ottawa, Canada). Many thanks to Tobias Bauer (Karlsruhe, Germany), Gerhard M. Tarmann (Hall, Austria) and an anonymous colleague for the review of the submitted version of the paper and their constructive comments. 


\section{References}

Gallon, R.C. (2001) Revision of the Ceratogyrus spp. formerly included in Coelogenium (Araneae: Theraphosidae, Harpactirinae). Mygalomorph, 2 (1), 1-20.

Gallon, R.C. (2010) On some southern African Harpactirinae, with notes on the eumenophorines Pelinobius muticus Karsch, 1885 and Monocentropella Strand, 1907 (Araneae, Theraphosidae). Bulletin of the British Arachnological Society, 15 (2), $29-48$. https://doi.org/10.13156/arac.2010.15.2.29

Gurney, A.B., Kramer, J.P. \& Steyskal, G.C. (1964) Some techniques for the preparation, study, and storage in microvials of insect genitalia. Annals of the Entomological Society of America, 57, 240-242. https://doi.org/10.1093/aesa/57.2.240

Haselböck, A., Schilling, A.-K., Wendt, I. \& Holstein, J. (2018) Alternative Methode zur manuellen Fixierung von flüssigkonservierten Arthropoden für die makroskopisch-fotografische Dokumentation. Arachne, 23 (1), 13-17.

Montesanto, G. (2015) A fast GNU method to draw accurate scientific illustrations for taxonomy. ZooKeys, 515, $191-206$. https://doi.org/10.3897/zookeys.515.9459

Müller, S. (2021) Gattungsbestimmung afrikanischer Vogelspinnen: Das Erstellen eines interaktiven Bestimmungsschlüssels für Theraphosidae mithilfe der Diversity Workbench. Bachelor Thesis. University Stuttgart. [unknown pagination]

Padial, J.M., Miralles, A., De la Riva, I. \& Vences, M. (2010) The integrative future of taxonomy. Frontiers in Zoology, 7 (1), 16. https://doi.org/10.1186/1742-9994-7-16

Rajaei, H., Gelbrecht, J., Schulz, N., \& Hausmann, A. (2021) Minoa lutea Schwingenschuss, 1954 (Lepidoptera: Geometridae: Larentiinae) recognized as bona species. Zootaxa, 4903 (2), 255-264. https://doi.org/10.11646/zootaxa.4903.2.5

Robinson, G.S. (1976) The preparation of slides of Lepidoptera genitalia with special reference to the Microlepidoptera. Entomologist's Gazette, 27, 127-132.

Su, Y.N. (2016) A simple and quick method of displaying liquid-preserved morphological structures for microphotography. Zootaxa, 4208 (6), 592-593. https://doi.org/10.11646/zootaxa.4208.6.6

Ungureanu, E.M. (1972) Methods for dissecting dry insects and insects preserved in fixative solutions or by refrigeration. Bulletin World Health Organization, 47 (2), 239-244.

Wanke, D., Bigalk, S., Krogmann, L., Wendt, I. \& Rajaei, H. (2019) The Fixator-A simple method for mounting of arthropod specimens and photography of complex structures in liquid. Zootaxa, 4657 (2), 385-391. https://doi.org/10.11646/zootaxa.4657.2.11

Wanke, D. \& Rajaei, H. (2018) An effective method for the close up photography of insect genitalia during dissection: a case study on the Lepidoptera. Nota Lepidopterologica, 41 (2), 219-223. https://doi.org/10.3897/nl.41.27831

Wanke, D., Hausmann, A., Krogmann, L., Petrányi, G. \& Rajaei, H. (2020) Taxonomic revision of the genus Nychiodes Lederer, 1853 (Geometridae: Ennominae: Boarmiini) with description of three new species - an integrative approach. Zootaxa, 4812 (1), 001-061. https://doi.org/10.11646/zootaxa.4812.1.1

Wanke, D., Hausmann, A., Sihvonen, P., Krogmann, L. \& Rajaei, H. (2020) Integrative taxonomic review of the genus Synopsia Hübner, 1825 in the Middle East (Lepidoptera: Geometridae: Ennominae). Zootaxa, 4885 (1), 027-050. https://doi.org/10.11646/zootaxa.4885.1.2 